(1)

CrossMark

\title{
IL-17F, rather than IL-17A, underlies airway inflammation in a steroid-insensitive toluene diisocyanate-induced asthma model
}

\author{
Rongchang Chen ${ }^{1,5}$, Qingling Zhang ${ }^{1,5}$, Shuyu Chen ${ }^{2,5}$, Haixiong Tang ${ }^{3,5}$, \\ Peikai Huang ${ }^{1}$, Shushan Wei ${ }^{1}$, Zhenyu Liang ${ }^{1}$, Xin Chen $^{4}$, Ailin Tao $^{2}$ and \\ Lihong Yao ${ }^{1}$
}

\begin{abstract}
Affiliations: 'State Key Laboratory of Respiratory Disease, Guangzhou Institute of Respiratory Health, The First Affiliated Hospital of Guangzhou Medical University, Guangzhou Medical University, Guangzhou, China. ${ }^{2}$ Guangdong Provincial Key Laboratory of Allergy and Clinical Immunology, State Key Laboratory of Respiratory Disease, The Second Affiliated Hospital of Guangzhou Medical University, Guangzhou Medical University, Guangzhou, China. ${ }^{3}$ Dept of Respiratory Medicine, Minzu Hospital of Guangxi Zhuang Autonomous Region, Guangxi Medical University, Nanning, China. ${ }^{4}$ Dept of Respiratory Medicine, Zhujiang Hospital, Southern Medical University, Guangzhou, China. ${ }^{5}$ These authors contributed equally to this work.
\end{abstract}

Correspondence: Lihong Yao, State Key Laboratory of Respiratory Disease, Guangzhou Medical University, No. 195, West Dongfeng Road, 510180 Guangzhou, China. E-mail: yaolh4321d126.com

@ERSpublications

In a TDI-induced steroid-insensitive murine asthma model, IL-17A restricts allergic responses through suppressing Th2 inflammation and eosinophil recruitment, while IL-17F modulates airway inflammation by driving Th17 response and neutrophil infiltrates http://ow.ly/vP2z30nk7Z3

Cite this article as: Chen R, Zhang Q, Chen S, et al. IL-17F, rather than IL-17A, underlies airway inflammation in a steroid-insensitive toluene diisocyanate-induced asthma model. Eur Respir J 2019; 53: 1801510 [https://doi.org/10.1183/13993003.01510-2018].

ABSTRACT Steroid insensitivity constitutes a major problem for asthma management. Toluene diisocyanate (TDI) is one of the leading allergens of asthma that induces both T-helper Th2 and Th17 responses, and is often associated with poor responsiveness to steroid treatment in the clinic.

We sought to evaluate the effects of inhaled and systemic steroids on a TDI-induced asthma model and to find how interleukin (IL)-17A and IL-17F function in this model. BALB/c mice were exposed to TDI for generating an asthma model and were treated with inhaled fluticasone propionate, systemic prednisone, anti-IL-17A, anti-IL-17F, recombinant IL-17A or IL-17F.

Both fluticasone propionate and prednisone showed no effects on TDI-induced airway hyperresponsiveness (AHR), bronchial neutrophilia and eosinophilia, and epithelial goblet cell metaplasia. TDI-induced Th2 and Th17 signatures were not suppressed by fluticasone propionate or prednisone. Treatment with anti-IL-17A after TDI exposure led to increased AHR, aggravated mucus production and airway eosinophil recruitment, accompanied by amplified Th2 responses, whereas anti-IL-17F ameliorated TDI-induced AHR and airway neutrophilia, with decreased Th17 responses. Recombinant IL-17A and IL-17F showed opposite effects to the monoclonal antibodies.

IL-17A and IL-17F exert distinct biological effects during airway inflammation of a TDI-induced asthma model, which is unresponsive to both inhaled and systemic steroids.

This article has supplementary material available from erj.ersjournals.com

Received: Aug 082018 | Accepted after revision: Jan 062019

Copyright OERS 2019 


\section{Introduction}

About $10-25 \%$ of adult-onset asthma is occupational associated, with diisocyanates (including toluene diisocyanate (TDI) and methylene diphenyl diisocyanate) the most commonly reported causes [1], which often responds poorly to steroid treatment, leading to poor prognosis even after cessation of exposure [2]. A deeper understanding of the disease is urgently needed to find more therapeutic targets. TDI-induced asthma is often characterised by accumulation of a large number of neutrophils and a smaller number of eosinophils in the airways [3]. Multiple mechanisms are thought to be involved in the induction of TDI-induced asthma, including immunological, genetic, neurogenic, etc., leading to the definition of two subtypes of TDI-induced asthma: immunological and nonimmunological [4]. Evidence from human and animal models suggests that different subgroups of $\mathrm{CD} 4^{+}$T-cells (mainly T-helper Th1, Th2 and Th17 cells), together with their secreted cytokines, critically contribute to TDI-induced asthma [4], and therefore further studies are needed to uncover their complex functions.

During the past decade, the role of interleukin (IL)-17 in immune and allergic disorders has been attracting increasing attention. IL-17 is a family of cytokines mainly produced by Th17 cells and consists of six members: IL-17A, IL-17B, IL-17C, IL-17D, IL-17E (also known as IL-25) and IL-17F [5]. Among them, IL-17A and IL-17F share the closest amino acid sequence identity and function through IL-17 receptors. Recent clinical and animal studies support the idea that IL-17 is critically involved in asthma pathogenesis. Elevated IL-17 concentrations were found in the airways of allergic asthma patients [6], and correlate positively with asthma severity and steroid insensitivity [7]. Inhibition of IL-17 using gene knockout mice or blocking antibody reduces ovalbumin (OVA)-induced airway hyperresponsiveness (AHR), bronchial inflammatory cell infiltration as well as airway vascular remodelling [8-10]. However, SCHNYDER-CANDRIAN et al. [11] discovered that exogenous IL-17 inhibited OVA-induced pulmonary eosinophil recruitment, Th2 inflammation and bronchial hyperreactivity. These opposite findings revealed that IL-17 conveys dual effects, which may be attributed to the conflicting biological functions of individual IL-17 family members, especially for IL-17A and IL-17F [12]. Researchers have already demonstrated an increased level of IL-17 in TDI-induced asthma. Anti-IL-17 neutralising antibody could decrease TDI-induced airway inflammation and AHR [13, 14], suggesting important roles of IL-17 in TDI-induced asthma. However, the separate functions of different subtypes of IL-17 have not been assessed.

We have previously established a TDI-induced asthma model with pronounced airway neutrophilia and eosinophilia. The aims of this study were 1) to evaluate the effects of inhaled and systemic steroids on TDI-induced asthmatic responses, and 2) to examine the roles of IL-17A and IL-17F in TDI-induced asthma.

\section{Methods}

For detailed methods including animal protocols and experimental procedures, see the supplementary material.

\section{Ethics statement}

All animal experiments described here complied with the guidelines of the Committee on the Use and Care of Animals of Guangzhou Medical University (Guangzhou, China), and were approved by the Animal Subjects Committee of Guangzhou Medical University.

\section{Statistics}

Data are expressed as mean with standard deviation. Results were interpreted using one-way ANOVA and Bonferroni's difference post hoc test with SPSS version 22.0 (SPSS, Chicago, IL, USA). Differences were considered statistically significant when $\mathrm{p}<0.05$.

\section{Results}

TDI-induced AHR and pathological changes were not prevented by inhaled fluticasone propionate or systemic prednisone

Allergic airway inflammation was induced by exposing BALB/c mice to TDI. As expected, this led to significant neutrophil and eosinophil accumulation with structural and functional abnormalities of the airways. Despite their known effects in mild and moderate asthma, corticosteroids do not seem to be of any help in our TDI-induced asthma model. Intraperitoneal injection of $5 \mathrm{mg} \cdot \mathrm{kg}^{-1}$ prednisone or intranasal instillation of $300 \mu \mathrm{g} \cdot \mathrm{kg}^{-1}$ fluticasone propionate once daily beginning from the first inhalation for a period of 1 week did inhibit TDI-induced airway mucus production as assessed by Periodic acid-Schiff staining and Muc5ac mRNA expression (figure 1a, d and e), yet TDI-induced airway inflammation, epithelial hyperplasia, AHR and smooth muscle thickening did not change after prednisone or fluticasone propionate treatment (figure $1 \mathrm{a}-\mathrm{c}$ and $\mathrm{f}$ ). Similarly, neither inhaled fluticasone propionate nor systemic prednisone affected the increased numbers of neutrophils and eosinophils in bronchoalveolar lavage fluid (BALF) of TDI-sensitised 
a)
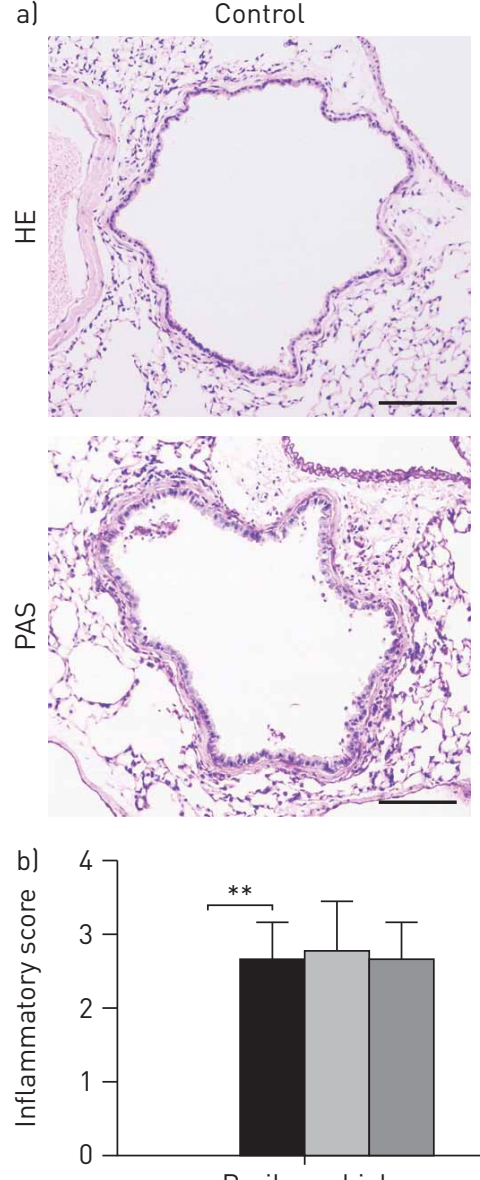

Peribronchial
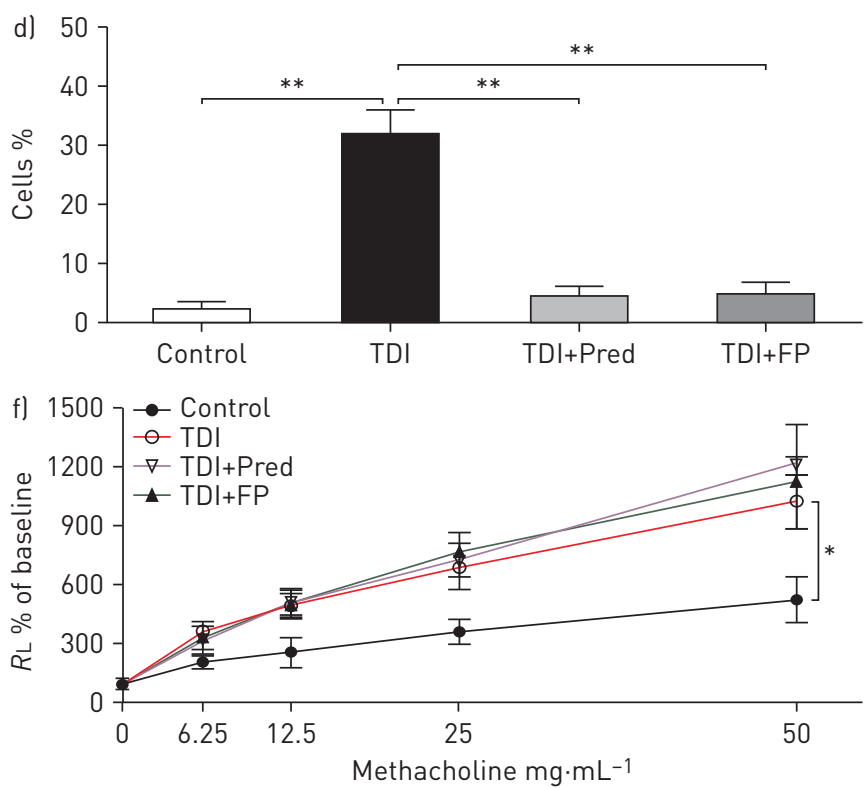

TDI+Pred
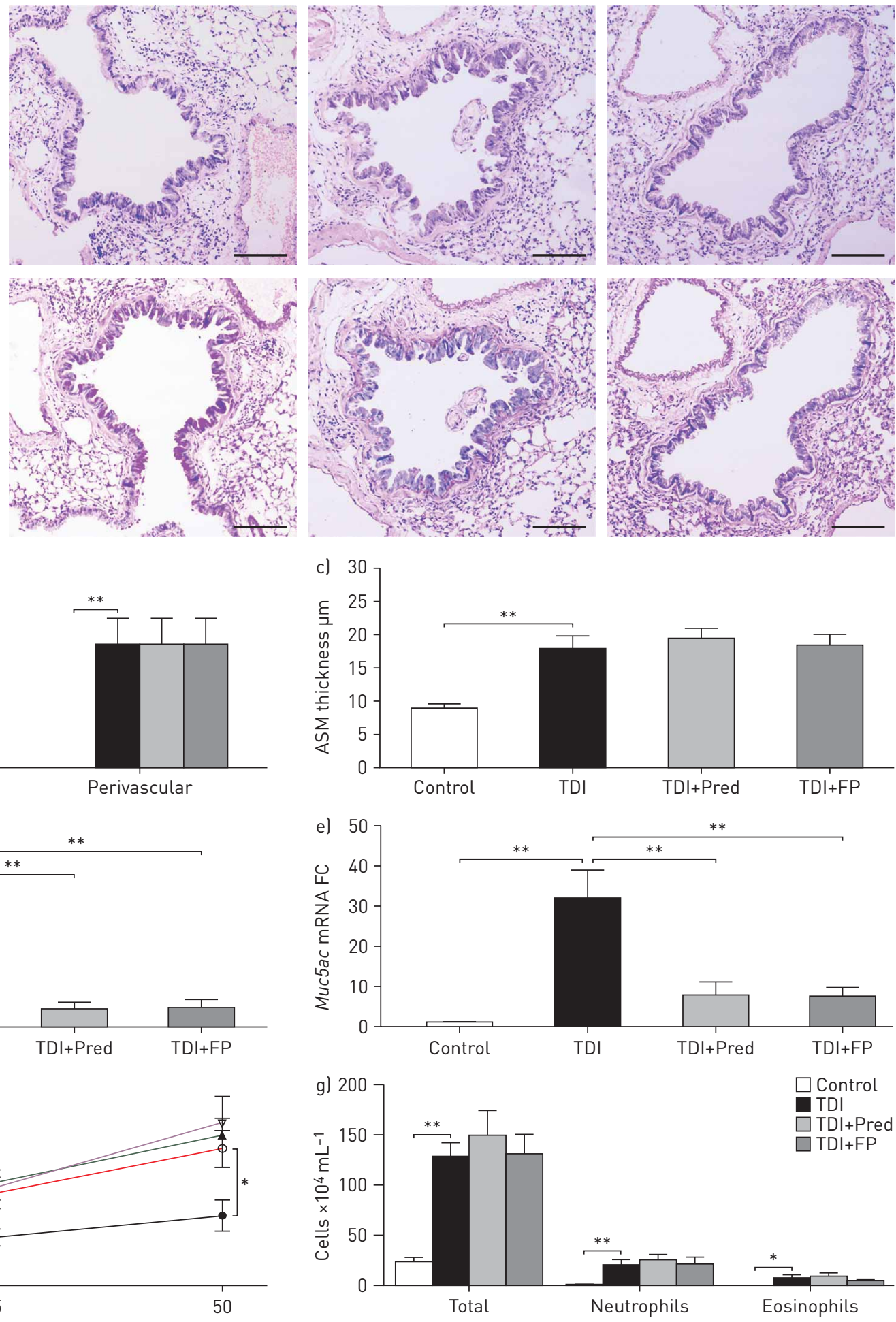

FIGURE 1 Inhaled fluticasone propionate (FP) or systemic prednisone (Pred) had no effects on toluene diisocyanate (TDI)-induced airway inflammation and bronchial hyperreactivity. HE: haematoxylin and eosin; PAS: Periodic acid-Schiff; ASM: airway smooth muscle; FC: fold change; $R$ L: lung resistance. a) Representative HE- and PAS-stained lung sections of different groups. Scale bar: $120 \mu \mathrm{m}$. b) Semiquantitative analysis of airway inflammation and cl analysis of ASM thickness. $n=8-10$ mice per group. d) Quantification of PAS-positive staining was determined by counting the number of PAS-positive epithelial cells. $n=8-10$ mice per group. e) Expression of Muc5ac (quantitative PCR) in the lung. $n=4$ mice per group. f) Airway hyperresponsiveness was measured by $R$ L. Results are shown as percentage of baseline value. $n=5$ mice per group. gl Numbers of total inflammatory cells, neutrophils and eosinophils. $n=8-10$ mice per group. ${ }^{*}: p<0.05 ;{ }^{* *}: p<0.01$. 
and challenged mice (figure $1 \mathrm{~g}$ ). In spite of this, greater numbers of neutrophils were seen in steroid-treated mice $\left(25.55 \pm 1.97 \times 10^{4} \mathrm{~mL}^{-1}\right.$ (TDI+prednisone group) versus $21.76 \pm 2.33 \times 10^{4} \mathrm{~mL}^{-1}$ (TDI+fluticasone propionate group) versus $20.20 \pm 2.13 \times 10^{4} \mathrm{~mL}^{-1}$ (TDI group); nonsignificant). Total serum IgE titres did not differ among all TDI-exposed groups (table 2).

\section{Both Th2 and Th17 responses are involved in TDI-induced asthma that cannot be suppressed by} inhaled fluticasone propionate or systemic prednisone

TDI-induced asthma is thought to be mediated by a mixed Th1, Th2 and Th17 response [13, 15]. Here, we set out to determine the percentages of those subtypes of T-helper cells in this asthma model and to evaluate the effect of steroid treatment. BALF and lung single-cell suspensions were harvested and stained for flow cytometry analysis. Results revealed that the percentages of CD4 interferon (IFN)- $\gamma^{+}$(Th1), CD4 IL- $4^{+}$(Th2) and CD4 IL-17A ${ }^{+}$(Th17) cells in BALF CD4 ${ }^{+}$cells of TDI-exposed mice were $4.60 \pm 0.65 \%$, $2.61 \pm 0.67 \%$ and $7.88 \pm 1.08 \%$ and the percentages in lung $\mathrm{CD} 4^{+}$cells were $0.97 \pm 0.21 \%\left(\mathrm{CD} 4 \mathrm{IFN}-\gamma^{+}\right)$, $0.61 \pm 0.10 \%\left(\mathrm{CD} 4 \mathrm{IL}-4^{+}\right)$and $1.99 \pm 0.39 \%\left(\mathrm{CD} 4 \mathrm{IL}-17 \mathrm{~A}^{+}\right.$) compared with $2.02 \pm 0.46 \%\left(\mathrm{CD} 4 \mathrm{IFN}-\gamma^{+}\right)$, $0.09 \pm 0.08 \%\left(\mathrm{CD} 4 \mathrm{IL}-4^{+}\right)$and $2.43 \pm 0.25 \%\left(\mathrm{CD} 4 \mathrm{IL}-17 \mathrm{~A}^{+}\right)$in BALF of control mice and $0.20 \pm 0.07 \%$ (CD4 IFN- $\left.\gamma^{+}\right), 0.11 \pm 0.02 \%\left(\mathrm{CD} 4 \mathrm{IL}-4^{+}\right)$and $0.73 \pm 0.10 \%\left(\mathrm{CD} 4 \mathrm{IL}_{-17 \mathrm{~A}^{+}}\right)$in lung $\mathrm{CD} 4^{+}$cells of control mice, whereas treatment with either inhaled fluticasone propionate or systemic prednisone had no effects on those TDI-induced T-helper cell subsets (table 1).

Th1-, Th2- and Th17-related cytokines were also quantified. Levels of Th2-related IL-4, IL-5 and IL-13 and Th17-related IL-17A and IL-17F in both BALF and lung homogenates were higher in TDI-sensitised and challenged mice compared with vehicle-exposed mice (table 2). The same was found for IL-6 and IL-1 $\beta$, which are critical for the differentiation and maturation of Th17 cells, although IL-1 $\beta$ was not detected in BALF of this model. Other cytokines, including IL-18, as well as the eosinophil chemoattractants CCL11 and CCL24, also significantly increased in TDI-induced asthma. Fluticasone propionate or prednisone blunted the release of IL- 5 but promoted IL-6 production in BALF, yet had no significant effects on the other cytokines (table 2). IFN- $\gamma$, IL-22 and IL-23 were not detected.

Th2 and Th17 signatures would result in distinct gene expression patterns in lung epithelia [16]. Thus, we assessed the relative expression of those genes in the whole lung. As shown in table 3, the mRNA levels of Th2 markers Ccl11 and Clca3 and Th17 markers Cxcl1, Cxcl3 and Csf3 were extensively upregulated by TDI. However, inhaled fluticasone propionate or systemic prednisone did not alter those gene expression patterns. The same was found for $I l 17 a$ and $I l 17 f$ mRNA expression.

\section{Blockade of IL-17A exacerbates TDI-induced airway inflammation}

As IL-17A and IL-17F were both increased in TDI-induced asthma, we wondered whether blocking each of them would help to ameliorate the disease. Monoclonal antibodies (100 $\mu \mathrm{g}$ per mouse) against IL-17A and IL-17F were administered to the mice after each airway challenge. Surprisingly, we observed completely different outcomes after neutralising IL-17A and IL-17F in TDI-exposed mice. Despite decreasing the level of secreted IL-17A in BALF, IL-17A monoclonal antibody exacerbated TDI-induced AHR and inflammation, led to more severe epithelial cell hyperplasia and remodelling, and drove greater numbers of eosinophils into the airway lumen (figure 2), coupled with markedly enhanced expression of eosinophil chemokine CCL24 in the lung (figure 3c). Administration of IL-17A monoclonal antibody did

\begin{tabular}{|c|c|c|c|c|}
\hline & Control group & TDI group & TDI+prednisone group & $\begin{array}{l}\text { TDI+fluticaso } \\
\text { propionate gro }\end{array}$ \\
\hline \multicolumn{5}{|l|}{ BALF } \\
\hline CD4 IFN- $\gamma^{+} \%$ & $2.02 \pm 0.46$ & $4.60 \pm 0.65^{* *}$ & $4.32 \pm 0.65$ & $4.34 \pm 1.19$ \\
\hline CD4 IL-4 $4^{+} \%$ & $0.09 \pm 0.08$ & $2.61 \pm 0.67^{* *}$ & $2.80 \pm 0.68$ & $2.73 \pm 0.52$ \\
\hline CD4 IL-17A $\%$ & $2.43 \pm 0.25$ & $7.88 \pm 1.08^{* *}$ & $8.24 \pm 0.92$ & $7.93 \pm 1.46$ \\
\hline \multicolumn{5}{|l|}{ Lung } \\
\hline CD4 IFN- $\gamma^{+} \%$ & $0.20 \pm 0.07$ & $0.97 \pm 0.21^{* *}$ & $1.05 \pm 0.24$ & $0.91 \pm 0.38$ \\
\hline CD4 IL- $-4^{+} \%$ & $0.11 \pm 0.02$ & $0.61 \pm 0.10^{* *}$ & $0.69 \pm 0.21$ & $0.91 \pm 0.16$ \\
\hline CD4 IL-17A $\%$ & $0.73 \pm 0.10$ & $1.99 \pm 0.39 * *$ & $1.79 \pm 0.25$ & $1.84 \pm 0.43$ \\
\hline
\end{tabular}

Data are presented as mean \pm SD. TDI: toluene diisocyanate; BALF: bronchoalveolar lavage fluid; IFN: interferon; IL: interleukin. ${ }^{* *}: \mathrm{p}<0.01$, compared with the control group. No significant differences were observed between the TDI group and the TDI+prednisone group or between the TDI group and the TDI+fluticasone propionate group. 
TABLE 2 Cytokine levels in steroid-treated mice

\begin{tabular}{|c|c|c|c|c|}
\hline & Control group & TDI group & TDI+prednisone group & TDI+fluticasone propionate group \\
\hline Lung IL-4 pg $\cdot \mathrm{mL}^{-1}$ & $5.15 \pm 1.10$ & $27.88 \pm 7.66^{*}$ & $21.64 \pm 3.05$ & $23.01 \pm 2.62$ \\
\hline Lung IL-5 $\mathrm{pg} \cdot \mathrm{mL}^{-1}$ & $1.29 \pm 3.37$ & $43.14 \pm 7.80 * *$ & $39.55 \pm 8.82$ & $43.88 \pm 6.46$ \\
\hline BALF IL-13 pg $\cdot \mathrm{mL}^{-1}$ & $2.39 \pm 0.48$ & $18.68 \pm 5.46^{* *}$ & $18.26 \pm 4.66$ & $19.25 \pm 4.17$ \\
\hline Lung IL-13 pg:mL ${ }^{-1}$ & $1.89 \pm 4.05$ & $74.68 \pm 15.80 * *$ & $58.91 \pm 12.05$ & $63.19 \pm 16.39$ \\
\hline BALF IL-17F $\mathrm{pg} \cdot \mathrm{mL}^{-1}$ & $0.00 \pm 0.00$ & $34.90 \pm 4.09 * *$ & $38.72 \pm 11.94$ & $41.78 \pm 22.52$ \\
\hline Lung IL-17F pg·mL ${ }^{-1}$ & $6.70 \pm 0.31$ & $37.32 \pm 10.50 *$ & $31.50 \pm 2.66$ & $30.48 \pm 2.83$ \\
\hline BALF IL-6 pg $\cdot \mathrm{mL}^{-1}$ & $8.76 \pm 2.20$ & $98.57 \pm 29.93^{* *}$ & $325.79 \pm 109.18^{\# \#}$ & $347.92 \pm 112.60^{\# \#}$ \\
\hline BALF IL-18 $\mathrm{pg} \cdot \mathrm{mL}^{-1}$ & $8.46 \pm 1.94$ & $310.75 \pm 98.40^{* *}$ & $325.75 \pm 40.97$ & $276.21 \pm 65.13$ \\
\hline BALF CCL11 $\mathrm{pg} \cdot \mathrm{mL}^{-1}$ & $8.31 \pm 2.02$ & $140.16 \pm 21.06^{* *}$ & $110.76 \pm 23.16$ & $106.23 \pm 37.58$ \\
\hline BALF CCL24 $\mathrm{pg} \cdot \mathrm{mL}^{-1}$ & $8.88 \pm 5.05$ & $223.81 \pm 47.17^{*}$ & $183.97 \pm 24.44$ & $201.24 \pm 14.32$ \\
\hline
\end{tabular}

Data are presented as mean \pm SD. TDI: toluene diisocyanate; BALF: bronchoalveolar lavage fluid; IL: interleukin. *: $p<0.05 ;{ }^{* *}$ : p<0.01, compared with the control group; ${ }^{\# \#}: p<0.01$, compared with the TDI group.

not inhibit the percentages of CD4 IL-17A ${ }^{+}$cells in BALF and lung, but gave rise to a number of CD4 IL- $4^{+}$cells (figure $3 \mathrm{a}$ and $\mathrm{b}$ ). At the same time, we detected larger amounts of Th2 cytokines IL- 4 and IL-5 in BALF after the mice were treated with IL-17A monoclonal antibody, whereas levels of IL-6, IL-18 and IL-1 $\beta$ in TDI asthmatic mice were not affected by IL-17A monoclonal antibody (figures $3 \mathrm{c}$ and $4 \mathrm{a}$ ). Accordingly, TDI-induced increased mRNA expression of Th2 markers Ccl11 and Clca3 was enhanced, in contrast with suppressed expression of Th17 markers Cxcl1, Cxcl3 and Csf3 (figure 4b). We found the opposite results after treating the TDI-sensitised and challenged mice with recombinant IL-17A (supplementary figures S2 and S3), except for the unaffected number of CD4 IL- $4^{+}$cells in BALF and lung despite their suppressed functions of secreting Th2 cytokines (supplementary figure S3a-c). These results suggest that IL-17A is restraining TDI-induced AHR and allergic responses.

Anti-IL-17F attenuates AHR and airway neutrophil inflammation in TDI-induced asthma

Blocking IL-17F produced a list of promising protective functions. Intraperitoneal injection of IL-17F monoclonal antibody at a dose of $100 \mu \mathrm{g}$ per mouse per time after each TDI challenge for a total of 3 times resulted in dramatically decreased airway inflammation and AHR, extensively compromised epithelial hyperplasia, goblet cell metaplasia and mucus production, as well as a significantly small number of neutrophils in BALF, while airway eosinophil recruitment was not inhibited (figure 2). In addition, the percentages of CD4 IFN- $\gamma^{+}, \mathrm{CD} 4 \mathrm{IL}-4^{+}$and CD4 IL-17A ${ }^{+}$cells in BALF and lung were also lowered after IL-17F monoclonal antibody treatment (figure $3 \mathrm{a}$ and b). Interestingly, IL-4, IL-5, IL-13, CCL11 and CCL24 in BALF or lung homogenates did not show obvious differences between mice treated with isotype

\begin{tabular}{|c|c|c|c|c|}
\hline & Control group & TDI group & TDI+prednisone group & TDI+fluticasone propionate group \\
\hline Ccl11 & $1.00 \pm 0.26$ & $7.85 \pm 2.22 * *$ & $7.21 \pm 2.27$ & $6.28 \pm 2.52$ \\
\hline Clca3 & $1.00 \pm 0.12$ & $41.45 \pm 8.52^{* *}$ & $47.30 \pm 11.95$ & $35.32 \pm 19.47$ \\
\hline Cxcl1 & $1.00 \pm 0.14$ & $8.37 \pm 2.99 * *$ & $7.57 \pm 2.54$ & $8.97 \pm 3.36$ \\
\hline Cxcl3 & $1.00 \pm 0.25$ & $4.30 \pm 1.17 * *$ & $7.06 \pm 3.64$ & $7.50 \pm 3.65$ \\
\hline Csf3 & $1.00 \pm 0.35$ & $18.15 \pm 4.22^{* *}$ & $32.99 \pm 12.71$ & $32.91 \pm 11.33$ \\
\hline II17a & $1.00 \pm 0.42$ & $28.71 \pm 4.68 * *$ & $33.59 \pm 5.78$ & $35.18 \pm 5.65$ \\
\hline II17f & $1.00 \pm 0.09$ & $2.61 \pm 0.72 *$ & $2.68 \pm 0.76$ & $2.12 \pm 0.46$ \\
\hline
\end{tabular}

mRNA expression was normalised to control values; data are presented as mean \pm SD fold change. *: $p<0.05$; $^{* *}: p<0.01$, compared with the control group. No significant differences were observed between the toluene diisocyanate (TDI) group and the TDI+prednisone group or between the TDI group and the TDI +fluticasone propionate group. 
a)
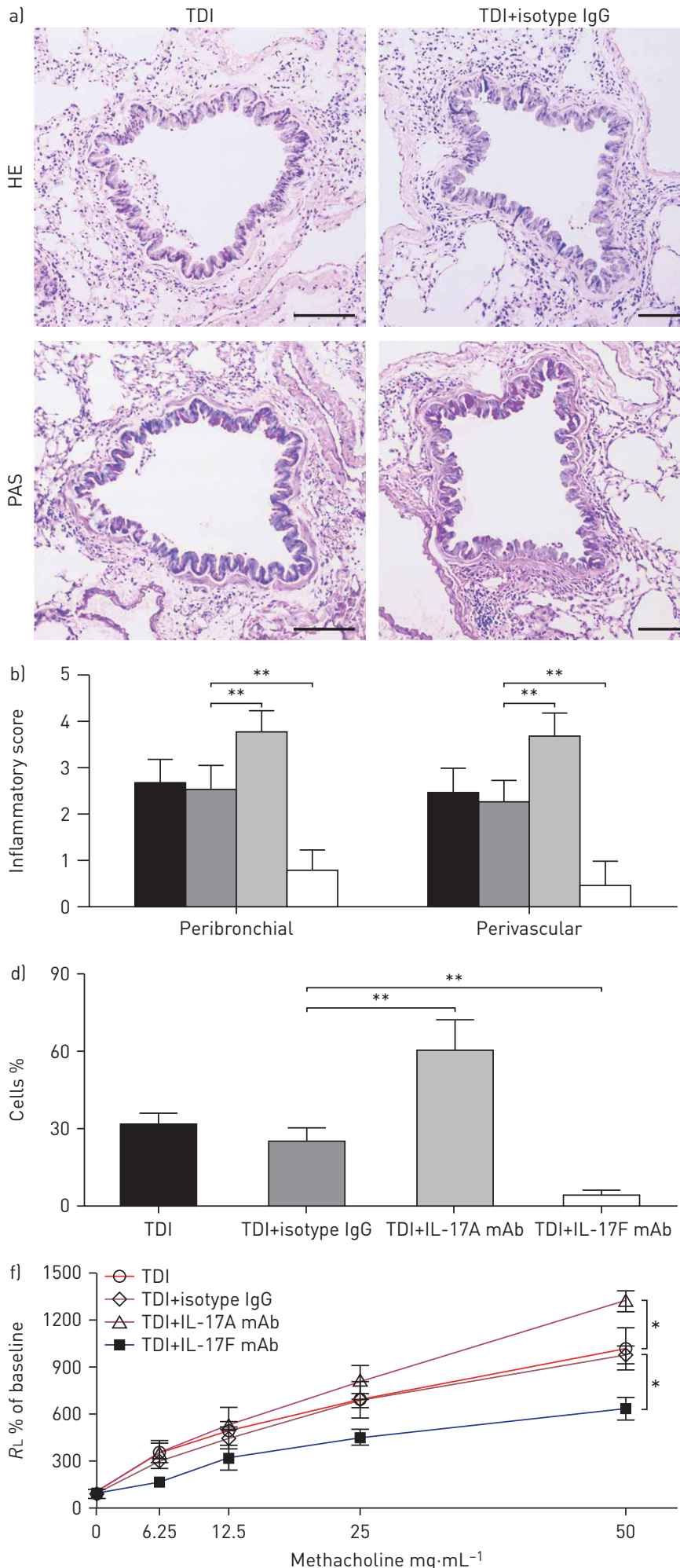

TDI+isotype $\lg G$
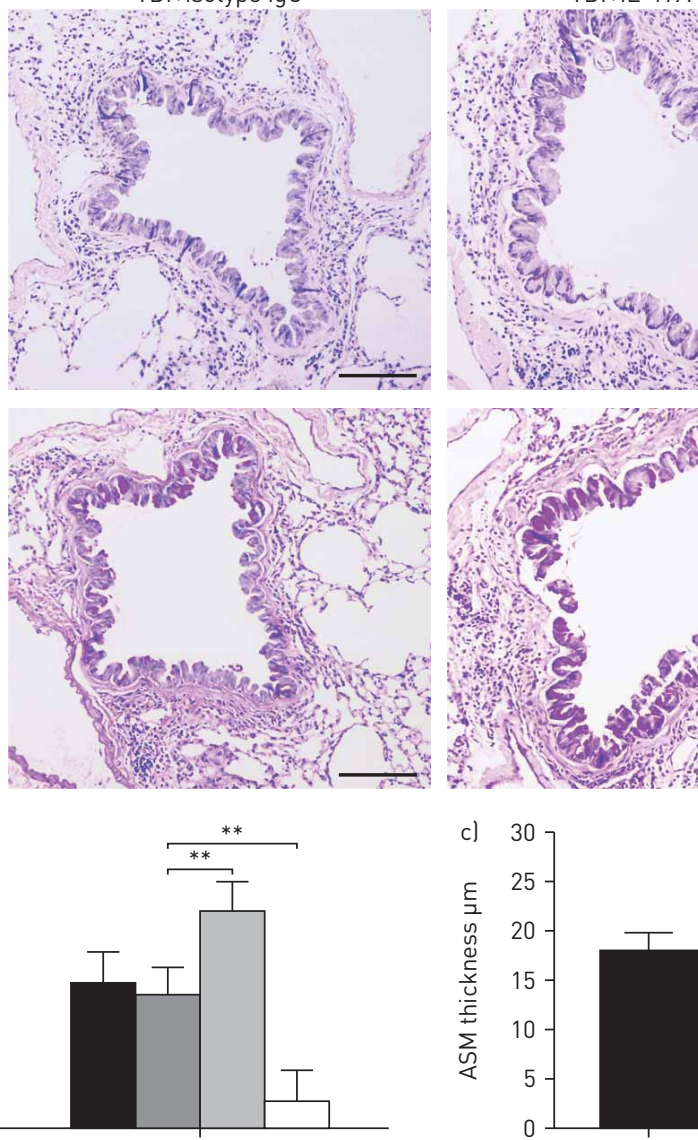

TDI

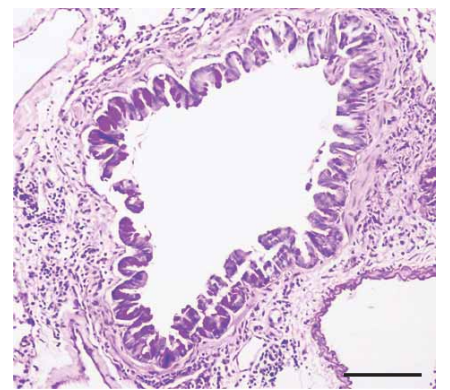

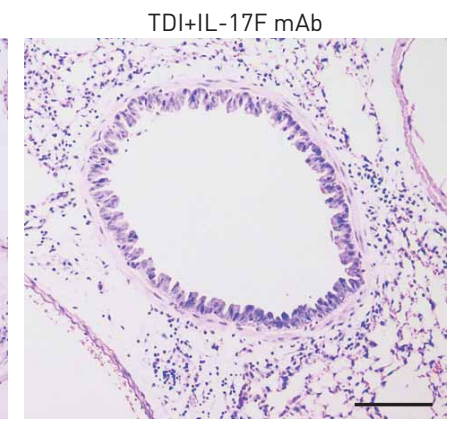

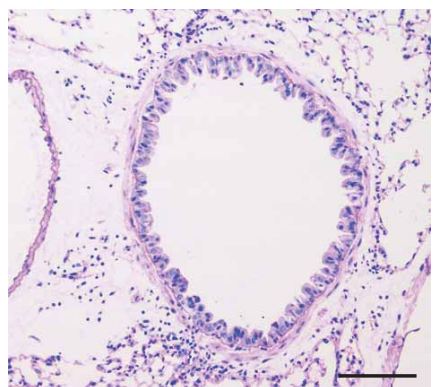

$* *$

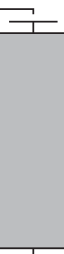

TDI+isotype IgG TDI+IL-17A mAb TDI+IL-17F mAb

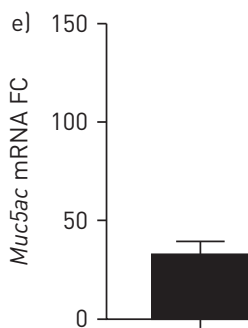

TDI

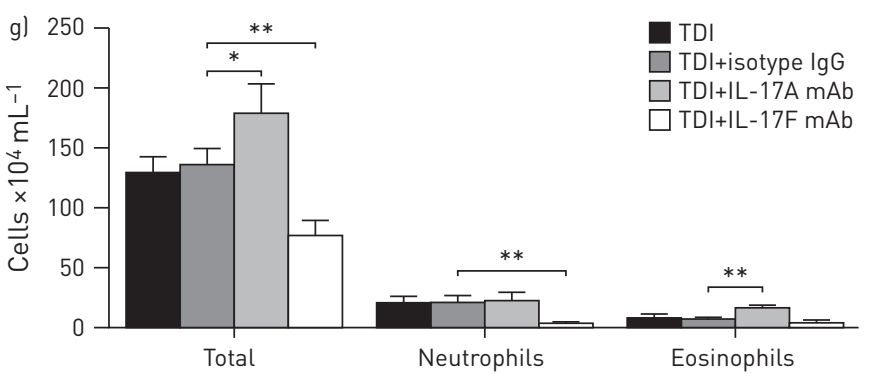

FIGURE 2 Interleukin (IL)-17A monoclonal antibody (mAb) and IL-17F mAb had opposite effects on toluene diisocyanate (TDI)-induced airway inflammation and bronchial hyperreactivity. HE: haematoxylin and eosin; PAS: Periodic acid-Schiff; ASM: airway smooth muscle; FC: fold change; $R \mathrm{~L}$ : lung resistance. a) Representative HE- and PAS-stained lung sections of different groups. Scale bar: $120 \mu \mathrm{m}$. b) Semiquantitative analysis of airway inflammation and c) analysis of ASM thickness. $n=8-10$ mice per group. d) Quantification of PAS-positive staining was determined by counting the number of PAS-positive epithelial cells. $n=8-10$ mice per group. e) Expression of Muc5ac (quantitative PCR) in the lung. $n=4$ mice per group. f) Airway hyperresponsiveness was measured by $R \mathrm{~L}$. Results are shown as percentage of baseline value. $\mathrm{n}=5$ mice per group. gl Numbers of total inflammatory cells, neutrophils and eosinophils. $n=8-10$ mice per group. ${ }^{*}: p<0.05 ;{ }^{* *}: p<0.01$. No significant differences were observed between the TDI group and the TDI+isotype IgG group. 

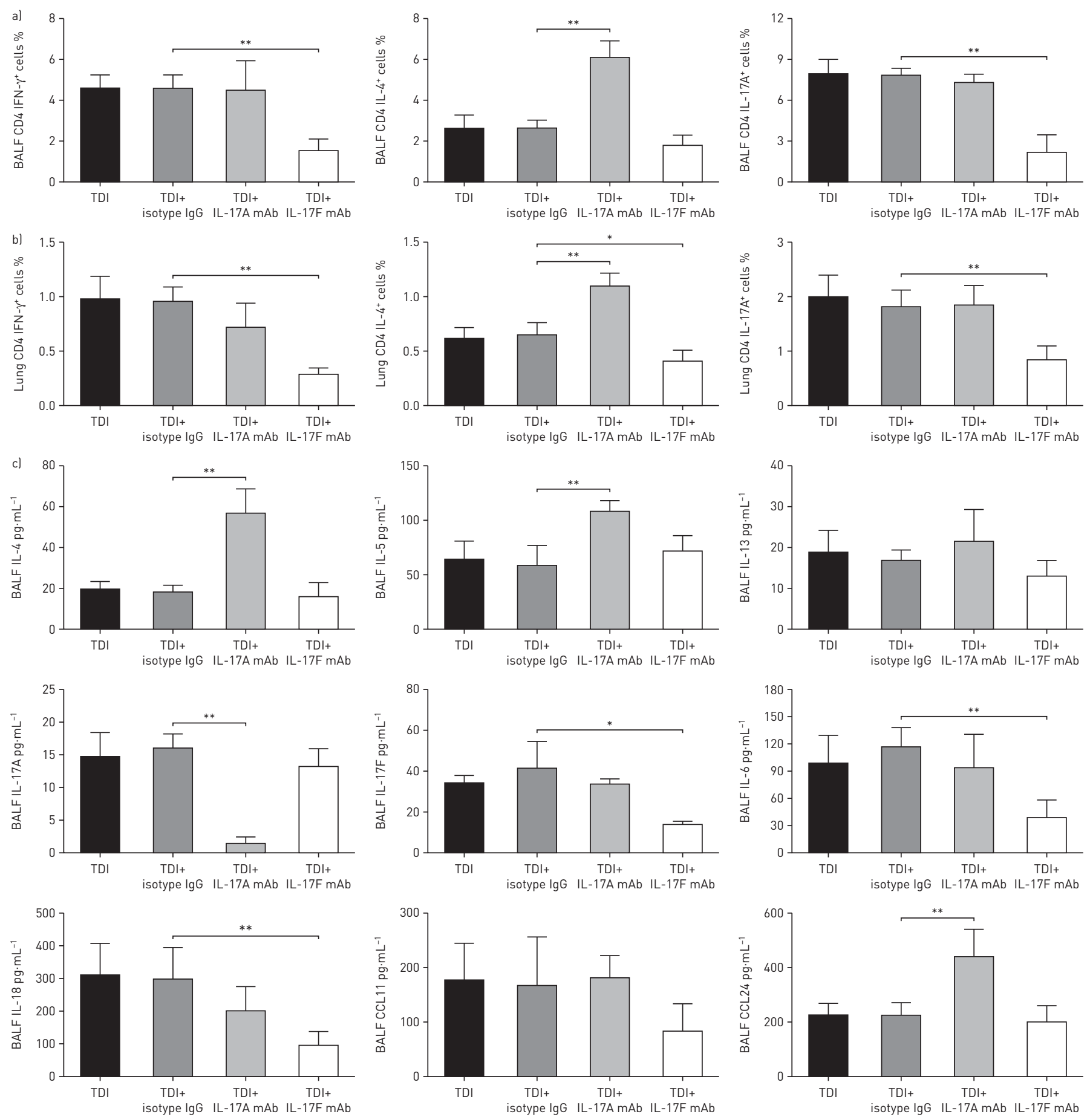

FIGURE 3 Blocking interleukin (IL)-17A and IL-17F displayed different capacity for orchestrating T-helper Th2 and Th17 responses. BALF:

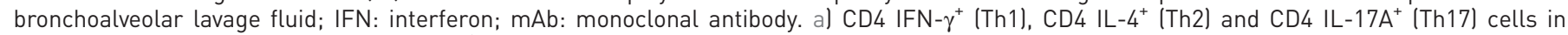
$\mathrm{CD} 4^{+}$cells of BALF. $\mathrm{n}=5$ mice per group. b) $\mathrm{CD} 4 \mathrm{IFN}-\gamma^{+}, \mathrm{CD} 4 \mathrm{IL}-4^{+}$and $\mathrm{CD} 4 \mathrm{IL}-17 \mathrm{~A}^{+}$cells in $\mathrm{CD} 4^{+}$cells of lung single-cell suspensions. $\mathrm{n}=5$ mice per group. c) IL-4, IL-5, IL-13, IL-17A, IL-17F, IL-6, IL-18, CCL11 and CCL24 levels in BALF were quantified by multiplex immunoassays or ELISA. $n=8-10$ mice per group. *: $p<0.05 ;{ }^{* *}: p<0.01$. No significant differences were observed between the TDI group and the TDI+isotype IgG group.

IgG and mice treated with IL-17F monoclonal antibody (figures $3 \mathrm{c}$ and $4 \mathrm{a}$ ), yet gene expression of Th17 markers Cxcl1, Cxcl3 and Csf3 was downregulated after neutralising IL-17F with monoclonal antibody in TDI-sensitised and challenged mice (figure 4b). At the same time, IL-17F monoclonal antibody treatment also inhibited the release of IL- 6 and IL-18 in BALF and IL-1 $\beta$ expression in lung (figures $3 c$ and $4 a$ ). Conversely, treatment with recombinant IL-17F aggravated the TDI-induced airway neutrophilic inflammation and Th17-related responses (supplementary figures S2 and S3), although AHR to methacholine was not significantly increased by IL-17F (supplementary figure S2f). 

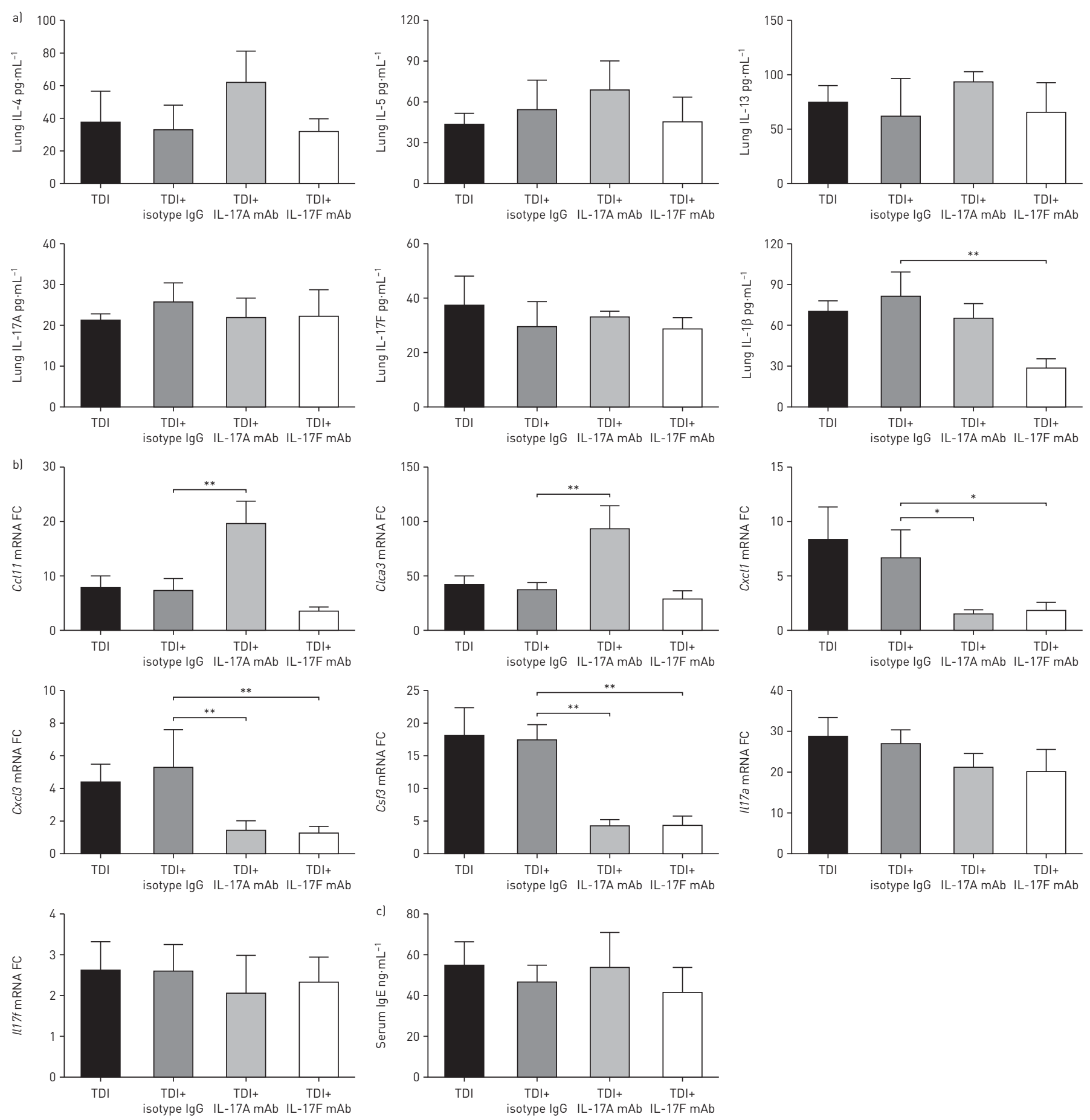

FIGURE 4 Neutralising interleukin (IL)-17A and IL-17F had different effects on toluene diisocyanate (TDI)-induced pulmonary expression of T-helper Th2 and Th17 markers. mAb: monoclonal antibody; FC: fold change. a) IL-4, IL-5, IL-13, IL-17A, IL-17F and IL-1 $\beta$ levels in whole lung homogenates were quantified by multiplex immunoassays or ELISA. $n=4$ mice per group. b) Whole lung tissue expression of Th2 markers Clca3 and $\mathrm{C} c l 11$ and Th17 markers Cxcl3, Cxcl1 and Csf3, as well as $1 / 17$ a and $1 / 17 f$, was assessed by quantitative PCR. n=4 mice per group. c) Total serum IgE concentrations were determined. $n=8-10$ mice per group. ${ }^{*}: p<0.05 ;{ }^{* *}: p<0.01$. No significant differences were observed between the TDI group and the TDI+isotype IgG group.

\section{Discussion}

In the present study, we assessed the effects of inhaled and systemic corticosteroids on TDI-induced asthmatic responses. Unexpectedly, both administration routes displayed few effects, indicating that we have identified a new steroid-insensitive asthma model that can be generated by a single allergen. In the meantime, we found for the first time that IL-17A restrains while IL-17F contributes to TDI-induced airway inflammation and AHR, demonstrating distinct roles for IL-17A and IL-17F. 
The family of diisocyanates consists of a list of highly reactive chemicals that have commonly been used in industrial production, including TDI, hexamethylene diisocyanate, methylene diphenyl diisocyanate, etc. They are all well-established important respiratory allergens and are connected with the majority of occupational asthma cases [17]. Here, we prepared a TDI-induced asthma model through dermal sensitisation followed by inhalation (supplementary material). Without neglecting the importance of respiratory sensitisation, the skin also acts as an important exposure route for initiating immune sensitisation. Skin exposure is well recognised as a mechanism for inducing immune sensitisation in TDI-induced asthma, including production of allergen-specific IgE [4]. Although the reported incidence of dermal exposure to TDI varies substantially [18], it predisposes the body with a very low concentration of TDI and is technically much easier.

As the mainstay therapy in asthma, corticosteroids display a great capacity for inhibiting allergic Th2 responses and inducing eosinophil apoptosis [19]. However, there are $\sim 10 \%$ of asthma patients that do not respond to steroid treatment, incurring a disproportionate amount of asthma-associated healthcare costs [20]. Studies have shown that the number of neutrophils correlates with poor responses to corticosteroids and decreasing lung function [21-23]. High numbers of neutrophils may reflect a non-Th2-dominated mechanism and, possibly, a steroid-resistant asthma phenotype, predicting more severe disease. Here, we used the TDI-induced asthma model (supplementary material). Allergic airway inflammation is dominated by accumulation of eosinophils and neutrophils, paralleled by increased numbers of Th2 and Th17 cells in both BALF and lungs of TDI-exposed mice. Compared with eosinophils, more neutrophils were seen in BALF of this model, together with predominant Th17 cells. Yet, to our surprise, treatment with inhaled fluticasone propionate $\left(300 \mu \mathrm{g} \cdot \mathrm{kg}^{-1}\right)$ or systemic prednisone $\left(5 \mathrm{mg} \cdot \mathrm{kg}^{-1}\right)$ failed to inhibit TDI-induced airway eosinophil infiltration and most of the Th2-related responses, although IL-5 and airway mucus production were actually blunted. At the same time, TDI-induced AHR, epithelial damage and hyperplasia, and Th17 signatures also showed no responsiveness to inhaled or systemic steroids. We even detected greater numbers of neutrophils (although not significant) in steroid-treated mice, accompanied by markedly higher levels of IL-6, an upstream cytokine for Th17 maturation, indicating that steroids not only exhibit no effects on TDI-induced eosinophilic inflammation, but also tend to propagate neutrophil inflammation and Th17 responses. Similar results were obtained when the TDI asthmatic mice were treated with systemic prednisone at a higher dose of $10 \mathrm{mg} \cdot \mathrm{kg}^{-1}$ (data not show). The results indicate that our TDI-induced asthma model is actually a steroid-insensitive asthma model, which agrees with findings in clinical patients $[2,24]$. However, this sounds contradictory to an earlier published paper showing potent anti-inflammatory effects of dexamethasone for TDI-induced eosinophilia [25]. Although it has been well recognised that eosinophils would undergo apoptosis after glucocorticoid treatment in asthma [19], steroid-resistant eosinophilic airway inflammation has also been described in both patients and animal models $[26,27]$. Further studies are needed to determine the reasons why eosinophils may have different responses to steroid treatment.

As one of the most important features of TDI-induced asthma, the large numbers of neutrophils accumulating in the airway make huge contributions to steroid resistance and greatly hamper control of the disease. Depletion of neutrophils could prevent TDI-induced AHR, epithelial damage and significantly reduce airway inflammation [28]. Therefore, targeting neutrophils might have potential therapeutic effects. Members of the IL-17 family, mainly IL-17A and IL-17F, are known to be potent neutrophil-mobilising chemokines [5]. They attract neutrophils on to mucosal surfaces by increasing the release of neutrophil-modulating cytokines and chemoattractants (including IL-1 $\beta$, IL-6, IL-8, granulocyte colony-stimulating factor, etc.) from epithelial and endothelial cells [29]. Genetic deletion or neutralisation of IL-17A diminishes neutrophil invasion and confers protection against organ injury and infection in mice $[30,31]$. IL-17A can also enhance neutrophil fungal killing functions and induce the production of reactive oxygen species [32]. Studies have already demonstrated an important role of IL-17 in TDI-induced asthma $[13,14]$ and we previously detected higher levels of IL-17A in this TDI-induced asthma model [33]. Thus, in the current study, we treated the TDI-sensitised and challenged mice with IL-17A monoclonal antibody, hoping to find some therapeutic effects. Unexpectedly, IL-17A antibody not only showed no effects on TDI-induced airway neutrophilic inflammation, but also augmented eosinophil aggregation, Th2-related responses, AHR, bronchial epithelial hyperplasia and mucus production, indicating that IL-17A functions to counteract eosinophilia and Th2 inflammation, which was verified a second time after we treated the TDI-inhaling mice with exogenous IL-17A. These findings appear to be in striking contrast to the aforementioned studies [13, 14] and differ from observations in OVA- or house dust mite (HDM)-induced asthma models [10, 34], but might help to account for the controversial dual roles of IL-17 (which consists of not only IL-17A) in allergic airway inflammation [8-11]. Consistent with our study, NAKAE et al. [8] and Hellings et al. [9] discovered that IL-17 deficiency or neutralisation significantly enhanced BALF IL-4 and IL-5, and aggravated OVA-induced eosinophilia. A recent study in a mouse model of allergic bronchopulmonary aspergillosis 
also supports the notion that antagonising IL-17A can boost allergen-induced airway eosinophilia and Th2 responses [12].

In contrast to the effects of IL-17A monoclonal antibody, IL-17F monoclonal antibody treatment had completely different outcomes. As mentioned earlier, IL-17F, another member of the IL-17 family, is also capable of driving neutrophilic inflammation [29]. With the greatest sequence homology ( $>50 \%)$ shared between IL-17A and IL-17F, few studies have disentangled the specific effects of these two IL-17 family members in pathological inflammation. Compared with IL-17A, IL-17F is much less studied. It was originally discovered in 2001 in bronchoalveolar lavage cells from asthma patients upon ragweed allergen challenge [35]. Later, in 2002, a Japanese team demonstrated that a coding region variant of the IL-17F gene is linked to chronic inflammatory airway diseases [36]. Recent studies proved that airway IL-17F expression positively correlates with the number of neutrophils and asthma severity $[37,38]$. In this study, the BALF IL-17F level in TDI-exposed mice was higher than in control mice. In line with the findings in the HDM-induced model [34], neutralising IL-17F with monoclonal antibody resulted in considerably diminished bronchial neutrophil infiltration, ameliorated airway epithelial injury and hyperplasia, decreased AHR, and blunted Th17-related responses, together with lower eosinophils (though not significant) in the airway. At the same time, IL-17F monoclonal antibody treatment also suppressed the excessive secretion of IL- 6 and IL-18 and the upregulated IL-1 $\beta$ expression in TDI asthmatic mice, all of which are critical mediators for neutrophil migration and activation [39]. The opposite results were seen when the TDI-sensitised and challenged mice were treated with recombinant IL-17F. These data suggest that IL-17F is the major culprit for TDI-induced neutrophilic inflammation and pathological changes.

In conclusion, we discovered a novel steroid-insensitive asthma model that is induced by TDI, providing an alternative approach for investigating mechanisms involved in severe asthma. In addition, we also found that IL-17A restricts TDI-induced allergic airway inflammation through counteracting Th2 responses and preventing eosinophil influx, while IL-17F contributes to TDI-induced airway neutrophilic inflammation and bronchial hyperresponsiveness, which might be effective therapeutic targets for severe asthma in the future.

Author contributions: L. Yao and H. Tang designed the experiment, analysed the results and wrote the manuscript. L. Yao, S. Chen and H. Tang performed the experiments. R. Chen, Q. Zhang, P. Huang and S. Wei helped with the experiment and data analysis. R. Chen and Q. Zhang helped to revise the manuscript. Z. Liang, X. Chen and A. Tao helped with data analysis and image processing. All the authors agreed upon the final approval of the version to be published, and to ensure questions relating to the accuracy or integrity of any part of the work are appropriately investigated and resolved.

Conflict of interest: None declared.

Support statement: This study was supported by National Postdoctoral Program for Innovative Talents (BX201700060), National Key R\&D Program of China (2017YFC1310601), National Natural Science Foundation of China (81800022), project funded by China Postdoctoral Science Foundation (2018M633034), Natural Science Foundation of Guangdong Province (2018A0303130244), Guangzhou Healthcare Collaborative Innovation Major Project (201604020012), Young Scientist Foundation of Guangxi Medical University (GXMUYSF201636), and Scientific Research Starting Foundation for Postdoctoral Fellows of Guangzhou. Funding information for this article has been deposited with the Crossref Funder Registry.

\section{References}

1 Gautrin D, Newman-Taylor AJ, Nordman H, et al. Controversies in epidemiology of occupational asthma. Eur Respir J 2003; 22: 551-559.

2 Paggiaro PL, Vagaggini B, Bacci E, et al. Prognosis of occupational asthma. Eur Respir J 1994; 7: 761-767.

3 Pollaris L, Devos F, De Vooght V, et al. Toluene diisocyanate and methylene diphenyl diisocyanate: asthmatic response and cross-reactivity in a mouse model. Arch Toxicol 2016; 90: 1709-1717.

4 Lummus ZL, Wisnewski AV, Bernstein DI. Pathogenesis and disease mechanisms of occupational asthma. Immunol Allergy Clin North Am 2011; 31: 699-716.

5 Isailovic $\mathrm{N}$, Daigo $\mathrm{K}$, Mantovani $\mathrm{A}$, et al. Interleukin-17 and innate immunity in infections and chronic inflammation. J Autoimmun 2015; 60: 1-11.

6 Molet S, Hamid Q, Davoine F, et al. IL-17 is increased in asthmatic airways and induces human bronchial fibroblasts to produce cytokines. J Allergy Clin Immunol 2001; 108: 430-438.

7 Chesné J, Braza F, Mahay G, et al. IL-17 in severe asthma. Where do we stand? Am J Respir Crit Care Med 2014; 190: 1094-1101.

8 Nakae S, Komiyama Y, Nambu A, et al. Antigen-specific T cell sensitization is impaired in IL-17-deficient mice, causing suppression of allergic cellular and humoral responses. Immunity 2002; 17: 375-387.

9 Hellings PW, Kasran A, Liu Z, et al. Interleukin-17 orchestrates the granulocyte influx into airways after allergen inhalation in a mouse model of allergic asthma. Am J Respir Cell Mol Biol 2003; 28: 42-50.

$10 \mathrm{Lu} \mathrm{S}, \mathrm{Li} \mathrm{H}, \mathrm{Gao}$ R, et al. IL-17A, but not IL-17F, is indispensable for airway vascular remodeling induced by exaggerated Th17 cell responses in prolonged ovalbumin-challenged mice. J Immunol 2015; 194: 3557-3566.

11 Schnyder-Candrian S, Togbe D, Couillin I, et al. Interleukin-17 is a negative regulator of established allergic asthma. J Exp Med 2006; 203: 2715-2725. 
12 De Luca A, Pariano M, Cellini B, et al. The IL-17F/IL-17RC axis promotes respiratory allergy in the proximal airways. Cell Rep 2017; 20: 1667-1680.

13 Kim SR, Lee KS, Park SJ, et al. PTEN down-regulates IL-17 expression in a murine model of toluene diisocyanate-induced airway disease. J Immunol 2007; 179: 6820-6829.

14 Liu SY, Wang WZ, Yen CL, et al. Leukocyte nicotinamide adenine dinucleotide phosphate-reduced oxidase is required for isocyanate-induced lung inflammation. J Allergy Clin Immunol 2011; 127: 1014-1023.

15 De Vooght V, Vanoirbeek JA, Haenen S, et al. Oropharyngeal aspiration: an alternative route for challenging in a mouse model of chemical-induced asthma. Toxicology 2009; 259: 84-89.

16 Choy DF, Hart KM, Borthwick LA, et al. $\mathrm{T}_{\mathrm{H}} 2$ and $\mathrm{T}_{\mathrm{H}} 17$ inflammatory pathways are reciprocally regulated in asthma. Sci Transl Med 2015; 7: 301ra129.

17 Kimber I, Dearman RJ, Basketter DA. Diisocyanates, occupational asthma and IgE antibody: implications for hazard characterization. J Appl Toxicol 2014; 34: 1073-1077.

18 Lynch HN, Prueitt RL, Goodman JE. Critique of the ACGIH 2016 derivation of toluene diisocyanate threshold limit values. Regul Toxicol Pharmacol 2018; 97: 189-196.

19 Woolley KL, Gibson PG, Carty K, et al. Eosinophil apoptosis and the resolution of airway inflammation in asthma. Am J Respir Crit Care Med 1996; 154: 237-243.

20 Trevor JL, Deshane JS. Refractory asthma: mechanisms, targets, and therapy. Allergy 2014; 69: 817-827.

21 Saffar AS, Ashdown H, Gounni AS. The molecular mechanisms of glucocorticoids-mediated neutrophil survival. Curr Drug Targets 2011; 12: 556-562.

22 Uddin M, Nong G, Ward J, et al. Prosurvival activity for airway neutrophils in severe asthma. Thorax 2010; 65: 684-689.

23 Chung KF, Wenzel SE, Brozek JL, et al. International ERS/ATS guidelines on definition, evaluation and treatment of severe asthma. Eur Respir J 2014; 43: 343-373.

24 Malo JL, Cartier A, Côté J, et al. Influence of inhaled steroids on recovery from occupational asthma after cessation of exposure: an 18-month double-blind crossover study. Am J Respir Crit Care Med 1996; 153: 953-960.

25 Song J, Zhao H, Dong H, et al. Mechanism of E-cadherin redistribution in bronchial airway epithelial cells in a TDI-induced asthma model. Toxicol Lett 2013; 220: 8-14.

26 American Thoracic Society. Proceedings of the ATS workshop on refractory asthma: current understanding, recommendations, and unanswered questions. Am J Respir Crit Care Med 2000; 162: 2341-2351.

27 Kim RY, Horvat JC, Pinkerton JW, et al. MicroRNA-21 drives severe, steroid-insensitive experimental asthma by amplifying phosphoinositide 3-kinase-mediated suppression of histone deacetylase 2. J Allergy Clin Immunol 2017; 139: 519-532.

28 De Vooght V, Smulders S, Haenen S, et al. Neutrophil and eosinophil granulocytes as key players in a mouse model of chemical-induced asthma. Toxicol Sci 2013; 131: 406-418.

29 Nembrini C, Marsland BJ, Kopf M. IL-17-producing T cells in lung immunity and inflammation. J Allergy Clin Immunol 2009; 123: 986-994.

30 Kono H, Fujii H, Ogiku M, et al. Role of IL-17A in neutrophil recruitment and hepatic injury after warm ischemia-reperfusion mice. J Immunol 2011; 187: 4818-4825.

31 Flannigan KL, Ngo VL, Geem D, et al. IL-17A-mediated neutrophil recruitment limits expansion of segmented filamentous bacteria. Mucosal Immunol 2017; 10: 673-684.

32 Taylor PR, Roy S, Leal SM Jr, et al. Activation of neutrophils by autocrine IL-17A-IL-17RC interactions during fungal infection is regulated by IL-6, IL-23, ROR $\gamma \mathrm{t}$ and dectin-2. Nat Immunol 2014; 15: 143-151.

33 Yao L, Zhao H, Tang H, et al. Chicken IgY facilitates allergic airway inflammation in a chemical-induced murine asthma model by potentiating IL-4 release. Toxicol Lett 2015; 239: 22-31.

34 Chenuet P, Fauconnier L, Madouri F, et al. Neutralization of either IL-17A or IL-17F is sufficient to inhibit house dust mite induced allergic asthma in mice. Clin Sci 2017; 131: 2533-2548.

35 Kawaguchi M, Onuchic LF, Li XD, et al. Identification of a novel cytokine, ML-1, and its expression in subjects with asthma. J Immunol 2001; 167: 4430-4435.

36 Hizawa N, Kawaguchi M, Huang SK, et al. Role of interleukin-17F in chronic inflammatory and allergic lung disease. Clin Exp Allergy 2006; 36: 1109-1114.

37 Sorbello V, Ciprandi G, Di Stefano A, et al. Nasal IL-17F is related to bronchial IL-17F/neutrophilia and exacerbations in stable atopic severe asthma. Allergy 2015; 70: 236-240.

38 Ricciardolo FLM, Sorbello V, Folino A, et al. Identification of IL-17F/frequent exacerbator endotype in asthma. J Allergy Clin Immunol 2017; 140: 395-406.

39 de Oliveira S, Rosowski EE, Huttenlocher A. Neutrophil migration in infection and wound repair: going forward in reverse. Nat Rev Immunol 2016; 16: 378-391. 